\title{
Development of a nine-IncRNA signature as a novel prognostic marker of estrogen receptor-negative breast cancer
}

\author{
ZHIWEI WANG $^{1 *}, \mathrm{JIE} \mathrm{WANG}^{1 *}, \mathrm{LEI} \mathrm{LIU}^{2}, \mathrm{QI} \mathrm{HE}^{1}$ and MIN WEI ${ }^{1}$ \\ ${ }^{1}$ Department of Breast Surgery, International Peace Maternity and Child Health Hospital, \\ Shanghai Jiao Tong University, Shanghai 200030; ${ }^{2}$ Department of Surgery, \\ The Affiliated Tumor Hospital of Nantong University, Nantong, Jiangsu 226361, P.R. China
}

Received June 27, 2019; Accepted November 12, 2019

DOI: $10.3892 / \mathrm{ol} .2020 .11391$

\begin{abstract}
Long non-coding RNAs (lncRNAs) have been demonstrated to be aberrantly expressed in several types of tumor, and dysregulated lncRNAs are suggested to play a prognostic role in breast cancer (BC). Estrogen receptor (ER) status is a prognostic factor in patients with ER-negative BC, which is associated with poor prognosis. Thus, the present study developed a prognostic lncRNA signature specifically for ER-negative BC, in order to predict the risk of post-surgery relapse and improve patient prognosis. A gene expression profile containing 1,631 lncRNAs was obtained by investigating and integrating publicly available cohorts of BC. Subsequently, a nine-lncRNA signature was developed and validated in two independent cohorts via the Cox regression model. Using the nine-lncRNA signature, patients in the discovery cohort were divided into high- and low-risk groups, with significantly different disease-free survival [DFS; hazard ratio $(\mathrm{HR})=2.718$, 95\% confidence interval $(\mathrm{CI})=2.115-3.494, \mathrm{P}<0.0001]$. Receiver operating characteristic curve analyses demonstrated that the area under the curve reached 0.908. Similar results were obtained in the two independent cohorts ( $\mathrm{HR}=1.499,95 \%$ $\mathrm{CI}=0.950-2.365, \mathrm{P}=0.04 ; \mathrm{HR}=1.262,95 \% \mathrm{CI}=1.056-1.510$,
\end{abstract}

Correspondence to: Dr Qi He or Dr Min Wei, Department of Breast Surgery, International Peace Maternity and Child Health Hospital, Shanghai Jiao Tong University, 910 HengShan Road, Shanghai 200030, P.R. China

E-mail: doc_hq@126.com

E-mail: minweiedu@163.com

\section{*Contributed equally}

Abbreviations: lncRNA, long non-coding RNAs; BC, breast cancer; ER, estrogen receptor; ROC, receiver operating characteristic; DEGs, differentially expressed genes; KEGG, Kyoto Encyclopedia of Genes and Genomes; DFS, disease-free survival; HR, hazard ratio; CI, confidence interval; GEO, Gene Expression Omnibus; FDR, false discovery rate

Key words: estrogen receptor-negative breast cancer, long non-coding RNA, bioinformatics analysis, prognostic markers
$\mathrm{P}=0.01$ ), respectively. Furthermore, the nine lncRNAs were demonstrated to play important roles in the cell invasion and metastasis of different types of tumor. The differentially expressed genes (DEGs) identified between the high- and low-risk groups were consistently high in the discovery and validation cohorts. Functional analysis indicated that these DEGs, as well as genes co-expressed with the nine lncRNAs, were involved in cancer-associated signaling pathways, all of which provide further evidence for the predictive ability of the nine-lncRNA signature. Overall, the present study developed a novel prognostic biomarker for ER-negative BC.

\section{Introduction}

Breast cancer (BC) is the one of the most common malignancies in women worldwide, and the leading cause of cancer-associated mortality (1). In 2018, 40,000 people died of breast cancer (2). Long-term survival rates decrease from 90 to $5 \%$ following the development of distant metastasis or recurrence, thus leading to poor prognosis in patients with BC (3). Tamoxifen monotherapy poses as an anti-estrogen agent in the mammary tissue and is considered effective in decreasing the recurrence rate and improving the overall survival (OS) of patients with BC (4). For patients with estrogen receptor (ER)-positive $\mathrm{BC}$, the response rate of tamoxifen monotherapy decreased by $50-70$ and $5-10 \%$ for patients with ER-negative BC. Thus, the development of novel prognostic biomarkers is critical in order to accurately assess the outcome and provide individualized treatment strategies for patients with ER-negative BC.

Long non-coding RNAs (lncRNAs) are defined as RNA transcripts $>200$ base pairs in length (5). Accumulating evidence demonstrates IncRNA dysregulation have in multiple human diseases, particularly different types of cancer (6-8), and their expression is associated with cancer development and metastasis (9). For example, lncRNA Inhibiting Metastasis is upregulated in BC and is associated the inhibition of cell proliferation and metastasis (10). Furthermore, lncRNA HOXA transcript induced by TGF $\beta$ plays important roles in regulating the epithelial-to-mesenchymal transition (EMT), invasion and metastasis of BC (11). Several lncRNA signatures have been developed for specific BC subtypes, based on the aberrant expression of these lncRNAs in BC (12). 
The results of these studies have demonstrated the potential role of lncRNAs as biomarkers for the clinical outcome of ER-negative BC $(13,14)$.

A number of prognosis-associated lncRNAs have been identified as effective biomarkers via systematic, and subsequently used as lncRNA signatures for the prognosis of BC (15). For example, Wang et al (16) developed an 11-lncRNA signature using Cox regression analysis. Furthermore, Sun et al (17) developed an eight-lncRNA signature based on a weighted co-expressed network and competing endogenous RNA. Li et al (15) developed a five-lncRNA signature using the microarray re-annotation method. The aforementioned studies predominantly focused on all of the $\mathrm{BC}$ subtypes or patients with triple negative $\mathrm{BC}$; however, very few focused on the identification of prognostic biomarkers for patients with ER-negative BC.

In order to develop an IncRNA signature for prognosis, the present study obtained samples from patients with ER-negative BC from multiple datasets archived in the Gene Expression Omnibus (GEO) database (https://www.ncbi.nlm. nih.gov/geo/). The nine-lncRNA signature was developed using the Cox regression model, and validated in independent cohorts using the lncRNA expression profiles obtained using the microarray re-annotation method. Functional analyses demonstrated that these signature lncRNAs play important roles in tumor development and metastasis, which provides further evidence for the predictive ability of the signature.

\section{Materials and methods}

Data acquisition and pre-processing. A total of three datasets, GSE21653 (18,19), GSE58812 (20) and GSE19615 (21) were retrieved from the GEO database and measured using the Affymetrix Human Genome U133 Plus 2.0 Array platform (https://www.ncbi.nlm.nih.gov/geo/query/acc. cgi?acc=GPL570). The GSE21653 dataset contained 266 BC samples, among which 110 were ER-negative BC samples and used as the discovery cohort. The GSE58812 and GSE19615 datasets contained 107 and 45 ER-negative BC samples, respectively, which were used as the validation cohorts. In the present study, the gene expression profiles (CEL. files) for each dataset, and the corresponding clinical information, were downloaded. The robust multichip average algorithm was used for background adjustment of the gene expression profiles. Each probe-set ID was mapped to its Entrez gene ID using the corresponding platform annotation files. In the case of multiple probes being mapped to the same gene, the expression value for the gene was summarized as the arithmetic mean value of the multiple probes. In the instance that a probe was mapped to multiple or no genes, this probe was deleted.

IncRNA probe re-annotation. Using the BLASTn tools (https://blast.ncbi.nlm.nih.gov/Blast.cgi?PROGRAM=blastn\& PAGE_TYPE=BlastSearch\&LINK_LOC=blasthome), the probe annotation sequences supported by Affymetrix were aligned to the human long non-coding transcript sequences and protein-coding transcript sequences from the GENCODE database (Release 30; https://www.gencodegenes.org/human/), respectively. The results of the sequence alignment were filtered as follows: Only the probes that matched to one long non-coding transcript were reserved, and each transcript should identically match with more than six probes. Gene expression profiles containing 15,942 mRNAs and 1,631 lncRNAs were obtained using the re-annotation method.

Survival analysis. The univariate Cox proportional hazards regression model was used to identify lncRNAs significantly associated with disease-free survival (DFS) of patients in the discovery cohort (22), with $\mathrm{P}<0.05$ as the threshold. Subsequently, the multivariate Cox proportional hazards regression model was used to obtain robust prognosis-associated IncRNAs, which were used to develop the risk-score model according to the following equation:

$$
\text { RiskScore }=\sum_{k=1}^{n} E x p_{k} * e^{H R}{ }_{k}
$$

Where $n$ is the number of prognostic lncRNAs, $\operatorname{Exp}_{k}$ is the expression value of the prognostic $\operatorname{lncRNAs}$ and $e^{H R}{ }_{k}$ is the estimated regression coefficient of the IncRNAs in the multivariate Cox regression analysis.

The present study used a stepwise regression method (23) in order to perform multivariate regression analysis on 16 IncRNAs, from which nine stable molecules were obtained. Stepwise regression is commonly used to introduce variables, one by one, into the model. Following each introduction of an explanatory variable, an F-test is performed, and the explanatory variables that have been selected are subjected to a $\mathrm{t}$-test, respectively. When an explanatory variable is no longer significant due to the introduction of a subsequent explanatory variable, the former is deleted in order to ensure that only the significant variables are included in the regression equation prior to the introduction of each new variable. This procedure is an iterative process until neither a significant explanatory variable is selected into the regression equation, nor an insignificant explanatory variable is removed from the regression equation, in order to ensure that the resulting set of explanatory variables is optimal. The present study used the $\mathrm{R}$ step function to implement the stepwise regression process.

Consistency evaluation of DEGs. The reproducibility of the DEGs identified in the different datasets (GSE21653, GSE58812 and GSE19615) was evaluated using consistency analysis. The concordance score was calculated as $\mathrm{k} / \mathrm{n} \times 100 \%$ for several DEG lists extracted separately randomly from two of the three datasets (GSE21653, GSE58812 and GSE19615) which shared $\mathrm{n}$ genes, of which $\mathrm{k}$ genes demonstrated the same deregulation directions (up- or down-regulation). The score evaluates the consistency of DEGs by randomly extracting two independent datasets from two of the three datasets (GSE21653, GSE58812 and GSE19615).

The probability of observing a concordance score of $\mathrm{k} / \mathrm{n}$ by chance was evaluated using the cumulative binomial distribution model as follows:

$$
p=1-\sum_{i=0}^{k-1}\left(\begin{array}{l}
n \\
i
\end{array}\right) P_{0}^{\mathrm{i}}\left(1-P_{0}\right)^{n-i}
$$

Where $P_{0}$ (here, 0.5 ) is the probability of one gene having the concordant association between the two lists of genes by chance. 
Kyoto encyclopedia of genes and genomes (KEGG) enrichment analysis. KEGG pathway enrichment analysis was performed using the R package clusterProfiler (24) for DEGs and genes associated with the signature lncRNAs, and visualized using the R package DOSE (25). For both analyses, $\mathrm{P}<0.05$ was considered to indicate a statistically significant difference.

Statistical analysis. The median risk-score evaluated in each dataset by the signature for ER-negative BC samples was used as the cut-off (median score=26.1) in order to classify these samples into high- and low-risk groups. Kaplan-Meier (KM) plots were generated in order to assess DFS, followed by the log-rank test for the statistical comparison of the two groups. The predictive performance of the signature was further assessed using the pROC package (version 1.15.3). All statistical analyses were performed using the R software package (version 3.1.2;).

\section{Results}

Identification of prognosis-associated lncRNAs. For the present study, the gene expression profiles and clinical follow-up data of datasets GSE21653,GSE58812 and GSE19615 were downloaded, resulting in a total of 262 ER-negative BC samples. Subsequently, gene expression profiles containing 15,942 mRNAs and 1,631 lncRNAs were obtained via the probe re-annotation method. The univariate Cox proportional hazards regression model identified 16 prognosis-associated lncRNAs in the GSE21653 dataset, among which five were protective factors and 11 were risk factors $(\mathrm{P}<0.05$; Table I). A number of IncRNAs have been reported to be associated with the invasion and metastasis of various types of cancer. For example, lncRNA Long Intergenic Non-Protein Coding RNA, P53 Induced Transcript (LINC-PINT) has been reported to inhibit cancer cell proliferation, invasion and migration in osteosarcoma by downregulating micro (miR)-21 (26). Furthermore, LINC-PINT is known to inhibit tumor cell invasion through a highly conserved sequence element (27). The IncRNA LINC00324 has been demonstrated to promote cell proliferation in gastric cancer by binding with human antigen $\mathrm{R}$ and stabilizing FAM83B expression (28). In addition, the lncRNA Homeobox A11 antisense (HOXA11-AS) regulates the JAK-STAT signaling pathway via the miR-15a-3p/STAT3 axis in order to promote liver cancer growth and metastasis (29).

Development of the nine-lncRNA signature. Using the multivariate Cox proportional hazards regression model, a total of nine lncRNAs were identified from the 16 DFS-associated IncRNAs with optimal predictive performance in the discovery cohort, which were defined as the nine-lncRNA signature. The multivariate regression results of the nine identified lncRNAs are presented in Table II. Subsequently, the risk-score equations were formulated as follows: Risk-score $=2.4651^{*} \exp (\mathrm{CPS} 1-\mathrm{IT} 1)+0.5640^{*} \exp (\mathrm{FOXD} 2-\mathrm{AS} 1)$ $+0.8631^{*} \exp \left(\right.$ HOXA11-AS) $1.4586^{*} \exp ($ LINC00324) + $0.8866^{*} \exp (\mathrm{CKMT} 2-\mathrm{AS} 1)-0.6698^{*} \exp (\mathrm{BISPR})+1.3965^{*} \exp$ $($ SNAP25-AS1 $)+1.1144^{*} \exp ($ LOC101929340 $)+0.4768^{*} \exp$ (LINC02544). The risk-score for each ER-negative BC sample
Table I. LncRNAs associated with risk of post-surgery recurrence in ER-negative breast cancer.

\begin{tabular}{|c|c|c|c|c|}
\hline lncRNA_ID & Symbol & Coefficient & HR & P-value \\
\hline 101929340 & LOC101929340 & 1.1931 & 3.2973 & $1.61 \times 10^{-04}$ \\
\hline 100131067 & CKMT2-AS1 & 0.9910 & 2.6939 & $1.77 \times 10^{-04}$ \\
\hline 378805 & LINC-PINT & -0.7932 & 0.4524 & $1.85 \times 10^{-04}$ \\
\hline 284029 & LINC00324 & -1.4682 & 0.2303 & $3.23 \times 10^{-04}$ \\
\hline 101929504 & LINC02544 & 0.6051 & 1.8314 & $4.35 \times 10^{-04}$ \\
\hline 221883 & HOXA11-AS & 1.5265 & 4.6020 & $6.36 \times 10^{-04}$ \\
\hline 105221694 & BISPR & -0.6952 & 0.4990 & $7.27 \times 10^{-04}$ \\
\hline 29034 & CPS1-IT1 & 3.6059 & 36.8160 & $8.83 \times 10^{-04}$ \\
\hline 102724105 & ZNF528-AS1 & 1.0762 & 2.9335 & $1.25 \times 10^{-03}$ \\
\hline 100507463 & PSMB8-AS1 & -0.4446 & 0.6411 & $1.80 \times 10^{-03}$ \\
\hline 727915 & AGBL1-AS1 & 1.0646 & 2.8996 & $1.87 \times 10^{-03}$ \\
\hline 100131208 & SNAP25-AS1 & 1.9705 & 7.1743 & $2.33 \times 10^{-03}$ \\
\hline 84793 & FOXD2-AS1 & 0.9001 & 2.4599 & $4.14 \times 10^{-03}$ \\
\hline 100506211 & MIR210HG & 0.5215 & 1.6845 & $4.40 \times 10^{-03}$ \\
\hline 114614 & MIR155HG & -0.5074 & 0.6020 & $4.56 \times 10^{-03}$ \\
\hline 284930 & LOC284930 & 0.6582 & 1.9314 & $4.92 \times 10^{-03}$ \\
\hline
\end{tabular}

lncRNA, long non-coding RNA; ER, estrogen receptor; HR, hazard ratio.

in the discovery cohort was calculated for the nine-lncRNA signature. Receiver operating characteristic (ROC) curve analysis demonstrated that the 3-year area under curve reached 0.908 (Fig. 1A). Samples were divided into high- and low-risk groups for the nine-lncRNAs signature, with the median risk-scores as threshold. The 53 patients in the high-risk group had significantly worse DFS compared with the 57 patients in the low-risk group [hazard ratio $(\mathrm{HR})=2.718,95 \%$ confidence interval $(\mathrm{CI})=2.115-3.494, \mathrm{P}<0.0001$; Fig. 1B].

Validation of the nine-lncRNA signature. Risk-scores for samples in the validation cohort of the GSE19615 dataset were calculated using the nine-lncRNA signature. Subsequently, 23 and 22 samples were divided into low- and high-risk groups, respectively, with the median risk-score as the threshold. The DFS of patients in the high-risk group was significantly worse than that of the patients in the low-risk group $(\mathrm{HR}=1.499,95 \% \mathrm{CI}=0.950-2.365, \mathrm{P}=0.025$; Fig. 2A). Similarly, in the validation cohort of the GSE58812 dataset, 54 and 53 samples were classified into low- and high-risk groups, respectively. The OS rate of patients in the high-risk group was significantly worse compared with patients in the low-risk group $(\mathrm{HR}=1.316,95 \% \mathrm{CI}=1.094-1.582, \mathrm{P}=0.0035$; Fig. 2B). Furthermore, patients in the high-risk group demonstrated significantly worse DFS than those in the low-risk group $(\mathrm{HR}=1.262,95 \% \mathrm{CI}=1.056-1.510, \mathrm{P}=0.015$; Fig. $2 \mathrm{C})$. Overall, the nine-lncRNA characteristic risk-score $\kappa$ had a good prognostic stratification in both the training set (GSE21653) and the independent validation sets.

Association between risk-score and the expression levels of the signature lncRNAs. The distribution of risk-scores for 
Table II. Multivariate Cox regression analysis of the nine lncRNAs.

\begin{tabular}{lrrrrrr}
\hline IncRNA & Coefficient & HR & $\mathrm{z}$ & P-value & Lower 0.95 & Upper 0.95 \\
\hline CPS1-IT1 & 2.465 & 11.765 & 2.286 & 0.022 & 1.421 & 97.373 \\
FOXD2-AS1 & 0.564 & 1.758 & 1.682 & 0.093 & 0.911 & 3.391 \\
HOXA11-AS & 0.863 & 2.371 & 1.577 & 0.115 & 0.811 & 6.931 \\
LINC00324 & -1.459 & 0.233 & -2.879 & 0.004 & 0.086 & 0.628 \\
CKMT2-AS1 & 0.887 & 2.427 & 2.768 & 0.006 & 1.295 & 4.546 \\
SNAP25-AS1 & 1.397 & 4.041 & 2.080 & 0.038 & 1.084 & 15.064 \\
LOC101929340 & 1.114 & 3.048 & 2.681 & 0.007 & 1.350 & 6.882 \\
LINC02544 & 0.477 & 1.611 & 2.345 & 0.019 & 1.081 & 2.400 \\
BISPR & -0.670 & 0.512 & -2.480 & 0.013 & 0.301 & 0.869 \\
\hline
\end{tabular}
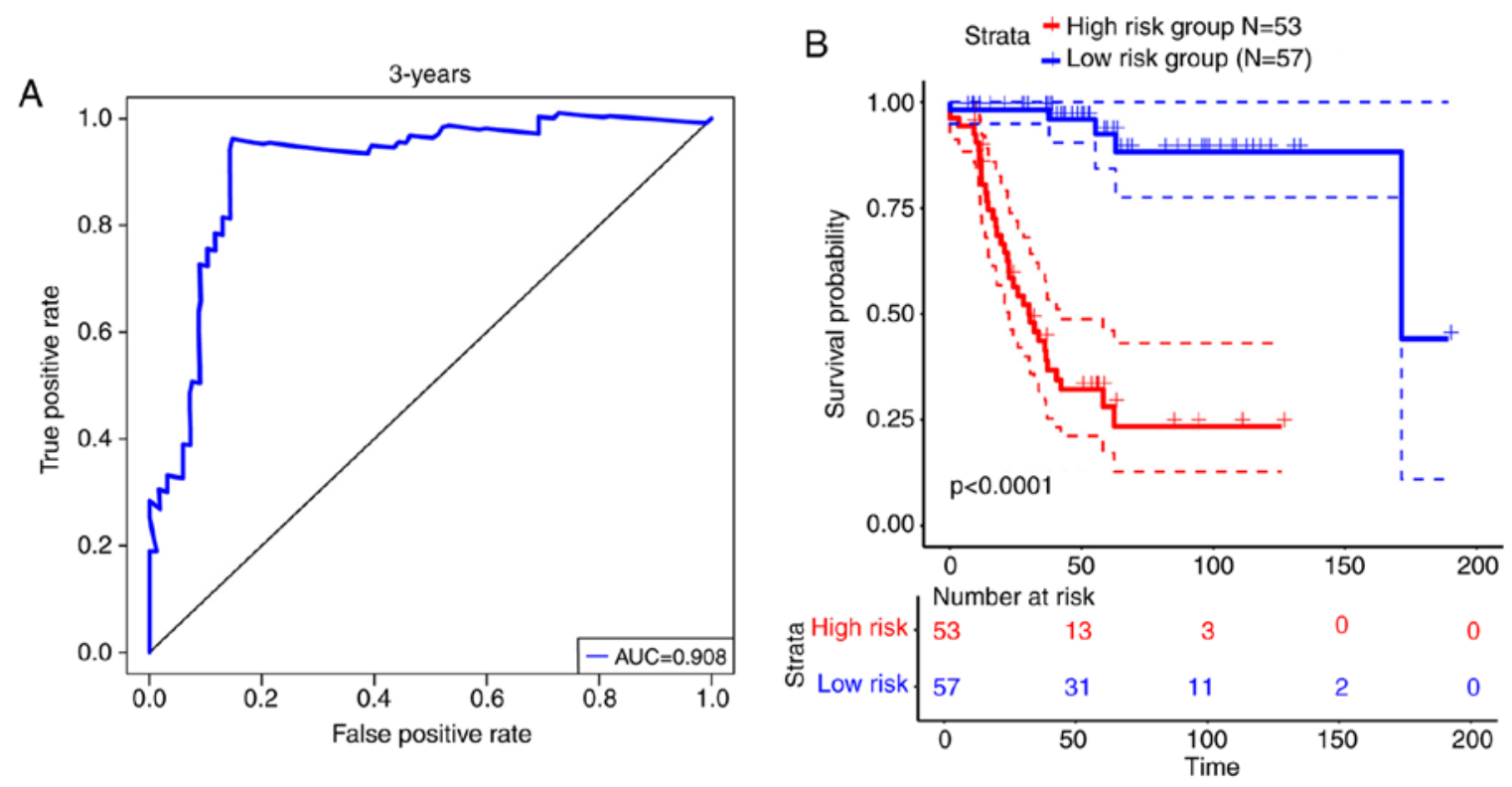

Figure 1. Prediction performance of the nine-lncRNA signature in the discovery cohort. (A) The 3-year AUC of the nine-lncRNA signature. (B) KM estimates of DFS for the high- and low-risk groups classified by the nine-lncRNA signature. Calibration lines on KM curves represent censored samples. IncRNA, long non-coding RNA; AUC, area under curve; DFS, disease-free survival; KM, Kaplan-Meier.

each patient calculated by the nine-lncRNA signature were analyzed. Together with patients' survival status, this identified a total of seven risk-associated lncRNAs (CKMT2-AS1, HOXA11-AS, CPS1-IT1, LOC101929340, FOXD2-AS1, SNAP25-AS1 and LINC02544), which were demonstrated to be overexpressed in patients with high risk-scores, and two protective lncRNAs (BISPRL and INC00324) were expressed at a lesser degree in patients with low risk-scores (Fig. 3A). Furthermore, eight of the nine lncRNAs were demonstrated to be significantly differentially expressed between the highand low-risk groups classified by the nine-lncRNAs signature (Fig. 3B, C and E-J); only HOXA11-AS exhibited marginally differential expression ( $\mathrm{P}=0.057$; Fig. 3D).

Association between the nine-lncRNA signature and clinical features. It has been reported that lymph node status, age, histodifferentiation, progesterone receptor (PR) status and human epidermal growth factor receptor 2 (HER-2) status may influence the prognosis of patients with $\mathrm{BC}(30,31)$. Using the multivariate Cox regression model, the present study demonstrated that the predictive performance of the nine-lncRNA signature was independent of the aforementioned clinical features. The results of the univariate and multivariate analyses for the nine-lncRNA signature and other clinical features are presented in Table III.

Nine-lncRNA signature-associated biological processes. The present study identified 1,276 DEGs between the 57 low-risk samples and the 53 high-risk samples classified by the nine-lncRNA signature in the discovery cohort (Table SI). As predicted, hierarchical clustering analysis of the top 30 most significant DEGs (Fig. 4A) demonstrated that samples in the high- and low-risk groups were divided into two clusters, respectively. Furthermore, KEGG pathway function analysis demonstrated that these DEGs were significantly enriched in 32 signaling pathways (Table SII), including signaling pathways associated with cancer cell growth, proliferation, migration and angiogenesis. The 
A

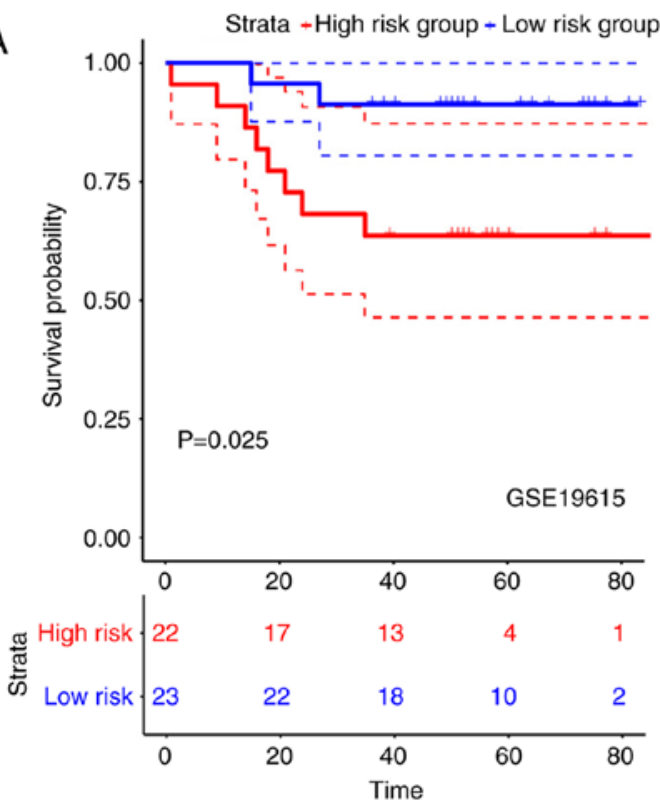

B

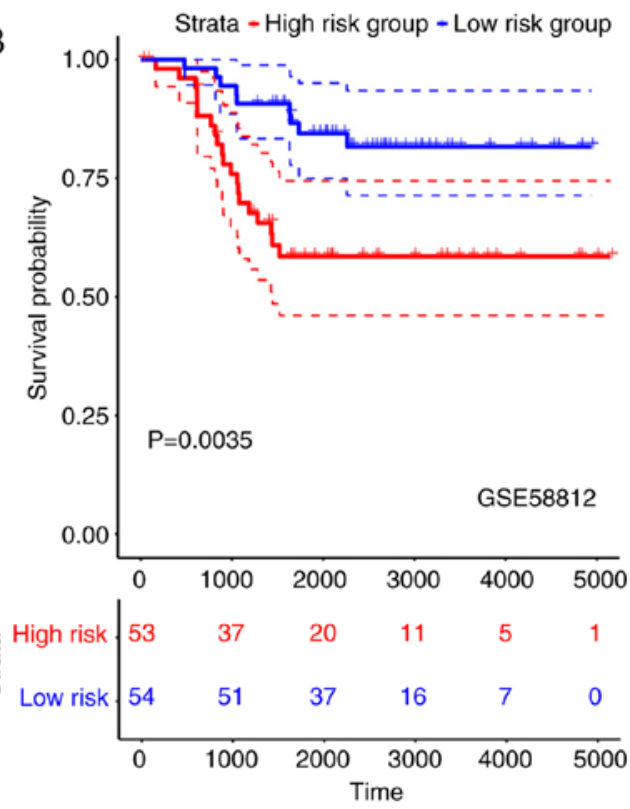

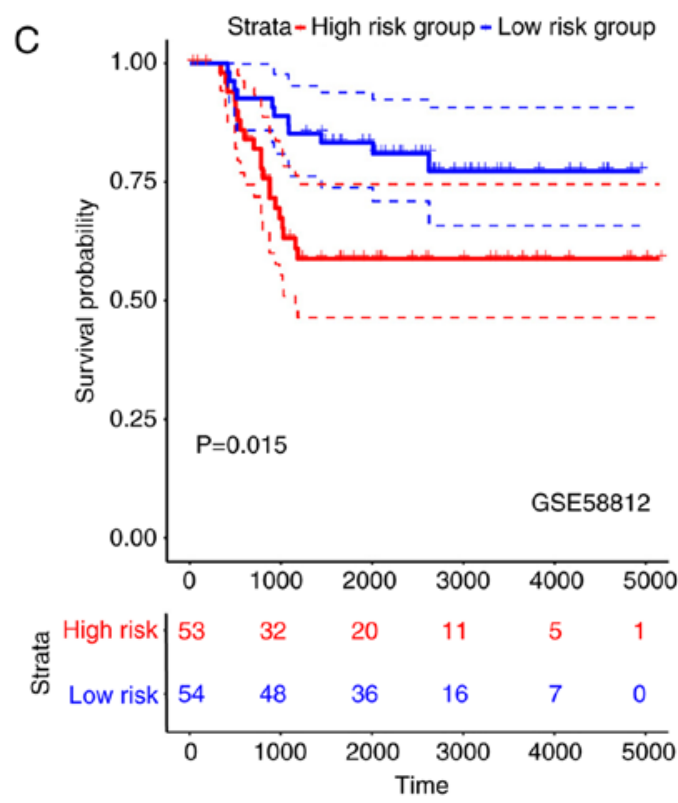

Figure 2. Validation of the nine-lncRNA signature. Efficiency of the nine-lncRNA signature for the risk stratification of patients with ER-negative BC was assessed in both the discovery and validation cohorts. (A) High risk group $n=22$, low risk group $n=23$. (B) High risk group $n=53$, low risk group $n=54$. (C) High risk group $n=53$, low risk group $n=54$. $\operatorname{lncRNA}$, long non-coding RNA; ER-negative $B C$, estrogen receptor-negative breast cancer.

associated signaling pathways in the present study included 'cytokine-cytokine receptor interaction' and 'cell adhesion molecules' (CAMs), and signaling pathways associated with the immune system, such as 'T-cell receptor signaling pathway', 'NF- $\kappa B$ signaling pathway', 'Th17 cell differentiation', and 'Th1 and Th2 cell differentiation'. Immune escape mechanisms have been reported to play an important role in the development and metastasis of cancer (32), which indicated the dysregulation of important carcinogenesis-associated biological processes between the classified high- and low-risk groups in the present study. With regards to the 110 samples assessed in the discovery cohort of the present study, the expression levels of 4,195 mRNAs were demonstrated to be significantly associated with the nine IncRNAs, among which 3,159 mRNA-lncRNA pairs were positively associated and 1,036 mRNA-lncRNA pairs were negatively associated (Pearson's correlation analysis, FDR $<0.01$ ) (Table SIII). These mRNAs were predicted to be co-expressed with the corresponding lncRNAs. The present study performed KEGG pathway function analysis using the co-expressed mRNAs, for each lncRNAs. A total of 37 KEGG pathways (Table SIV) were obtained for six lncRNAs (Fig. 4C), including cancer-associated signaling pathways, such as 'cell cycle', 'DNA replication' and 'p53 signaling pathway', and immune-associated signaling pathways including, 'Th1 and Th2 cell differentiation', 'B-cell receptor signaling pathway' and 'T-cell receptor signaling pathway'. Overall, the nine IncRNAs were associated with the carcinogenesis and development of patients with ER-negative BC. 

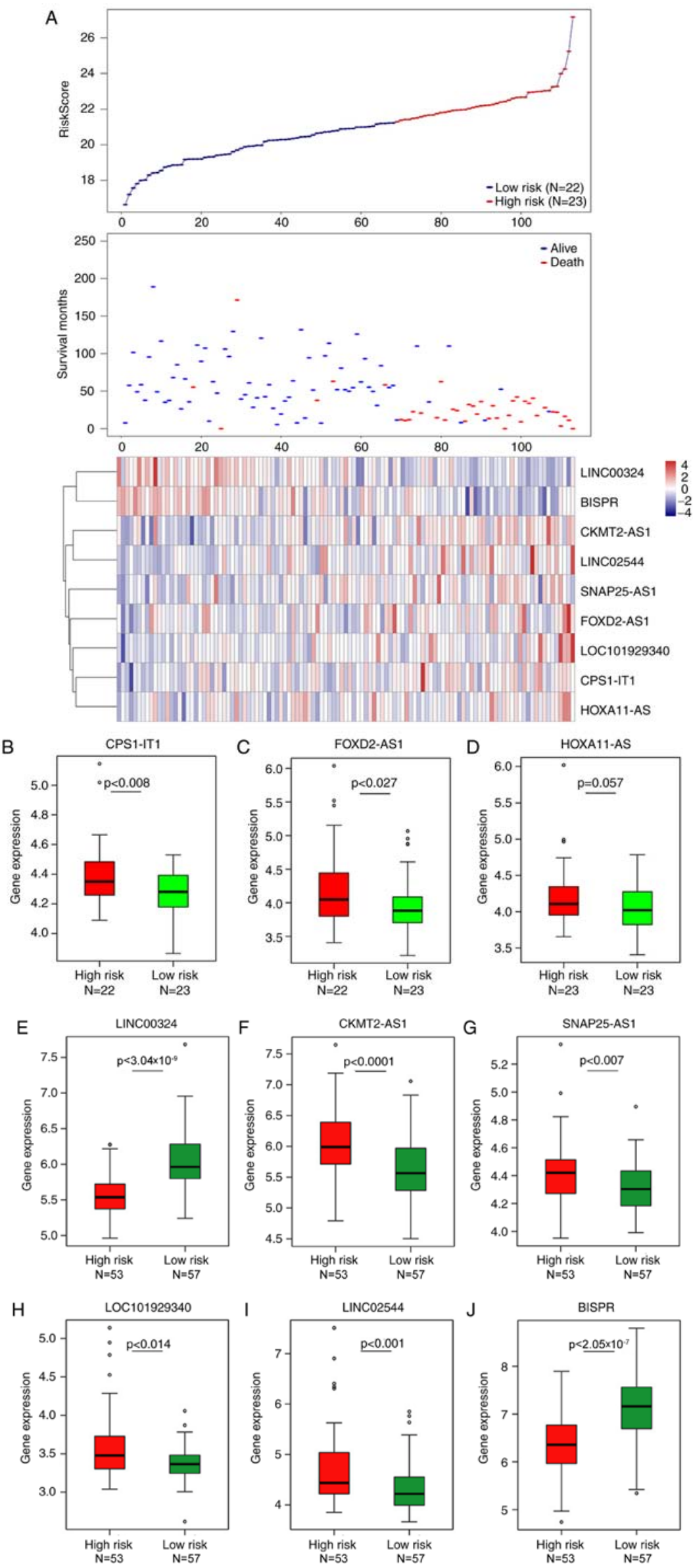

Figure 3. Gene expression analysis for the nine signature lncRNAs. (A) The distribution of risk-scores for each patient calculated using the nine-lncRNA signature (upper), survival status for each patient (middle) and the heatmap of expression levels for the nine lncRNAs (lower); High risk group $\mathrm{n}=23$, low risk group $n=22$. (B-J) Box-plots of the expression levels for the nine lncRNAs in the high- and low-risk groups. lncRNA, long non-coding RNA. 
Table III. Univariate and multivariate Cox regression analyses in the training set.

\begin{tabular}{|c|c|c|c|c|c|c|}
\hline \multirow[b]{2}{*}{ Factor } & \multirow[b]{2}{*}{ Sample, $\mathrm{n}$} & \multicolumn{2}{|c|}{ Univariate model } & \multicolumn{3}{|c|}{ Multivariate model } \\
\hline & & HR & P-value & HR & $95 \% \mathrm{CI}$ & P-value \\
\hline Nine-lncRNA risk score, high risk vs. low risk & 55 vs. 55 & 2.7180 & $5.7 \times 10^{-77}$ & 16.5578 & $4.908-55.854$ & $4.05 \mathrm{E}-06$ \\
\hline Stage, N1 vs. N0 & 50 vs. 58 & 2.9203 & 0.0011 & 2.6189 & $1.1417-6.007$ & 0.023 \\
\hline Grade, G3 vs G1 and G2 & 78 vs. 33 & 1.2295 & 0.0444 & 1.0540 & $0.7268-2.805$ & 0.874 \\
\hline Age, $\geq 50$ vs. $<50$ years & 70 vs. 40 & 1.1959 & 0.5981 & 1.0051 & $0.9761-1.035$ & 0.734 \\
\hline PR, Pos vs. Neg & 6 vs. 104 & 2.7364 & 0.0481 & 0.9480 & $0.2733-3.546$ & 0.981 \\
\hline HER-2, Pos vs. Neg & 15 vs. 89 & 1.3656 & 0.4579 & 1.2110 & $0.3727-3.935$ & 0.750 \\
\hline
\end{tabular}

lncRNA, long non-coding RNA; HR, hazard ratio; CI, confidence interval; lncRNA, long non-coding RNA; PR, progesterone receptor; HER-2, human epidermal growth factor receptor 2; Pos, positive; Neg, negative.
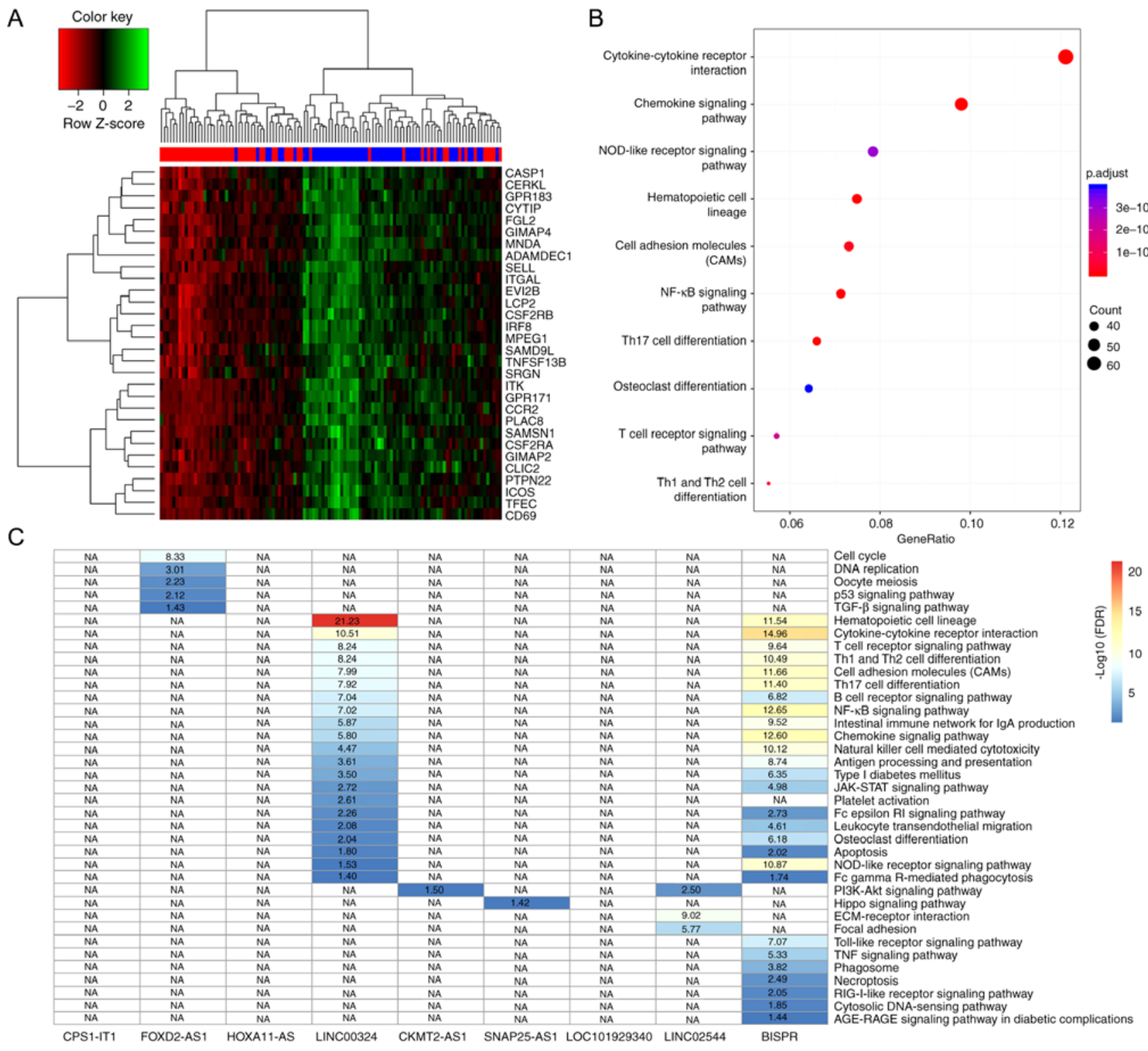

Figure 4. Function analysis for the nine-lncRNA signature. (A) Heatmap of the expression levels of the top 30 most significant DEGs between samples in the high- and low-risk groups. Red and blue refer to high- and low-risk samples, respectively. (B) The top 10 most significant KEGG pathways enriched by DEGs identified between high- and low-risk groups. X-axis represents the percentage of DEGs to pathway genes. The size of the dots represents the number of DEGs, and the color represents the significance level. (C) KEGG pathways enriched by genes co-expressed with lncRNAs. X-axis represents the nine lncRNAs. Color and number represents - $\log 10$ (FDR). IncRNA, long non-coding RNA; DEGs, differentially expressed genes; KEGG, Kyto Encyclopedia of Genes and Genomes; FDR, false discovery rate; NA; pathways insignificantly enriched by genes co-expressed with the corresponding lncRNAs. 


\section{Discussion}

In recent years, a number of studies have demonstrated that dysregulated lncRNAs play an important role in the carcinogenesis, development and metastasis of different types of tumor (33-35). A number of IncRNAs have been reported to be associated with the recurrence, metastasis and resistance to adjuvant therapy of patients with BC $(36,37)$. For example, lncRNA HOX Transcript Antisense Intergenic RNA has been demonstrated to enhance ER signaling and confer tamoxifen resistance in BC (38). Furthermore, upregulation of the lncRNA SRY-box 2 has been demonstrated to promote BC cell growth and invasion by activating the expression of the IncRNA Plasmacytoma variant translocation 1 (39). The degradation of the histone-lysine $\mathrm{N}$-methyltransferase EZH2, mediated by IncRNA Antidifferentiation noncoding RNA, has been indicated to decrease the invasion and metastasis of BC (40). With the development of genomic diagnostic tests such as Oncotype DX (41) and Coloprint (42), high-throughput gene expression profiles have become the principle method of identifying novel prognostic biomarkers of cancer. Although several lncRNA signatures have been developed for the prognosis of patients with BC $(43,44)$, there are currently none in use for clinical practice, particularly for ER-negative BC. Thus, the present study used publicly available gene expression profiles, together with the probe re-annotation method, in order to develop a DFS-associated nine-lncRNA signature, using the correlation analysis of the lncRNA expression data and clinical information from patients with ER-negative BC. The nine-lncRNA signature classified patients with ER-negative $\mathrm{BC}$ into high- and low-risk groups with significantly different DFS, which was validated in independent datasets. The results of the present study provide evidence for the repeatability and clinical value of the nine-lncRNA signature.

A number of signature IncRNAs have been reported to be associated with different types of tumor $(45,46)$. For example, CPS1 Intronic Transcript 1 has been demonstrated to suppress cell proliferation, invasion and metastasis in colorectal, ovarian, lung and liver cancer (28-31,47-50). Furthermore, upregulation of FOXD2 Adjacent Opposite Strand RNA 1 has been associated with poor prognosis in various types of tumor (51-53). HOXA11-AS has been indicated to promote BC invasion and metastasis by regulating EMT (54). Furthermore, LINC00324 acts as an oncogene involved in the tumorigenesis and progression of gastric and lung cancer (55). The lncRNA BST2 Interferon Stimulated Positive Regulator has been demonstrated to promote the progression of thyroid papillary carcinoma by regulating miR-21-5p (56). Despite numerous studies, the biological functions of several lncRNAs remain unclear, thus further research is required.

In the present study, DEGs between the high- and low-risk groups were identified in the discovery and validation cohorts, and high consistency scores were obtained between these cohorts (Table SV). KEGG pathway function analysis demonstrated that these DEGs were significantly enriched in signaling pathways associated with cancer cell growth, proliferation, migration and angiogenesis, such as 'cytokine-cytokine receptor interaction' and 'CAMs', and signaling pathways associated with the immune system, such as 'T-cell receptor signaling pathway', 'NF- $\kappa B$ signaling pathway', 'Th17 cell differentiation' and 'Th1 and Th2 cell differentiation'. Furthermore, genes co-expressed with these signature lncRNAs were significantly enriched in signaling pathways associated with cancer cell proliferation, migration and angiogenesis, such as 'cell cycle', 'DNA replication' and 'p53 signaling pathway', and signaling pathways associated with the immune system, such as 'Th1 and Th2 cell differentiation', 'B-cell receptor signaling pathway' and 'T-cell receptor signaling pathway'. Altogether, the nine lncRNAs assessed in the present study were associated with the carcinogenesis and development of tumors, which provides evidence for the prognostic ability of the nine-IncRNA signature.

Although candidate lncRNA biomarkers involved in tumorigenesis were identified by bioinformatics analysis, some limitations exist in the present study. Firstly, since clinical follow-up information was missing, certain factors, including the presence of patient's other health status, including ER, PR and HER2 expression, were not considered to determine prognostic biomarkers. Secondly, the signature obtained by bioinformatics analysis was insufficient for clinical practice and requires external experimental verification, such as in vitro/in vivo validation. Therefore, further work with complete clinical information and a larger sample size is required for future genetic and experimental studies.

Overall, the present study used the available microarray data and clinical information to develop a nine-IncRNA signature, in order to promote personalized treatment for patients with ER-negative BC. Functional analysis demonstrated that this signature was involved in several signaling pathways associated with cancer recurrence and metastasis, which provides evidence for the prediction of post-surgery relapse risk for patients with ER-negative BC. Large-scale prospective studies are required in the future, in order to further evaluate and validate the robustness of the signature prior to its clinical application. Furthermore, future studies are required in order to understand the molecular mechanisms underlying the nine-lncRNA signature.

\section{Acknowledgements}

Not applicable.

\section{Funding}

The present study was funded by the International Peace Maternity and Child Health Hospital (grant no. GFY 9307).

\section{Availability of data and materials}

The datasets used and/or analyzed during the present study are available from the corresponding author upon reasonable request.

\section{Authors' contributions}

ZW designed the present study. ZW, JW, LL and QH were responsible for acquisition, analysis and interpretation of data. $\mathrm{ZW}$ conducted the bioinformatics analyses. $\mathrm{ZW}$ and $\mathrm{QH}$ were responsible for drafting the manuscript and revising it critically. MW contributed to the conception of the present study and was responsible for approving the version to be published. All authors read and approved the final manuscript. 


\section{Ethics approval and consent to participate}

Not applicable.

\section{Patient consent for publication}

Not applicable.

\section{Competing interests}

The authors declare that they have no competing interests.

\section{References}

1. DeSantis C, Ma J, Bryan L and Jemal A: Breast cancer statistics, 2013. CA Cancer J Clin 64: 52-62, 2014.

2. Siegel RL, Miller KD and Jemal A: Cancer statistics, 2018. CA Cancer J Clin 68: 7-30, 2018.

3. Ma M, Huang W and Kong D: IL-17 inhibits the accumulation of myeloid-derived suppressor cells in breast cancer via activating STAT3. Int Immunopharmacol 59: 148-156, 2018.

4. Shandley LM, Spencer JB, Fothergill A, Mertens AC Manatunga A, Paplomata E and Howards PP: Impact of tamoxifen therapy on fertility in breast cancer survivors. Fertil Steril 107: 243-252.e245, 2017.

5. Spizzo R, Almeida MI, Colombatti A and Calin GA: Long non-coding RNAs and cancer: A new frontier of translational research? Oncogene 31: 4577-4587, 2012.

6. Sanchez Calle A, Kawamura Y, Yamamoto Y, Takeshita F and Ochiya T: Emerging roles of long non-coding RNA in cancer. Cancer Sci 109: 2093-2100, 2018.

7. Mitra SA, Mitra AP and Triche TJ: A central role for long non-coding RNA in cancer. Front Genet 3: 17, 2012.

8. Gibb EA, Vucic EA, Enfield KS, Stewart GL, Lonergan KM, Kennett JY, Becker-Santos DD, MacAulay CE, Lam S, Brown CJ and Lam WL: Human cancer long non-coding RNA transcriptomes. PLoS One 6: e25915, 2011.

9. Fatica A and Bozzoni I: Long non-coding RNAs: New players in cell differentiation and development. Nat Rev Genet 15: 7-21, 2014

10. Sas-Chen A, Aure MR, Leibovich L, Carvalho S, Enuka Y, Körner C, Polycarpou-Schwarz M, Lavi S, Nevo N, Kuznetsov Y, et al: LIMT is a novel metastasis inhibiting lncRNA suppressed by EGF and downregulated in aggressive breast cancer. EMBO Mol Med 8: 1052-1064, 2016.

11. Richards EJ, Zhang G, Li ZP, Permuth-Wey J, Challa S, Li Y, Kong W, Dan S, Bui MM, Coppola D, et al: Long non-coding RNAs (LncRNA) regulated by transforming growth factor (TGF) $\beta$ : LncRNA-hit-mediated TGF $\beta$-induced epithelial to mesenchymal transition in mammary epithelia. J Biol Chem 290: 6857-6867, 2015.

12. Zhao W, Luo J and Jiao S: Comprehensive characterization of cancer subtype associated long non-coding RNAs and their clinical implications. Sci Rep 4: 6591, 2014.

13. Lu SJ, Xie J, Li Y, Yu B, Ma Q and Liu BQ: Identification of lncRNAs-gene interactions in transcription regulation based on co-expression analysis of RNA-seq data. Math Biosci Eng 16 : 7112-7125, 2019

14. Bao S, Zhao H, Yuan J, Fan D, Zhang Z, Su J and Zhou M: Computational identification of mutator-derived lncRNA signatures of genome instability for improving the clinical outcome of cancers: A case study in breast cancer. Brief Bioinform, Oct 28 , 2019 (Epub ahead of print).

15. Li J, Wang W, Xia P, Wan L, Zhang L, Yu L, Wang L, Chen X, $\mathrm{X}$ iao $\mathrm{Y}$ and $\mathrm{Xu} \mathrm{C}$ : Identification of a five-lncRNA signature for predicting the risk of tumor recurrence in patients with breast cancer. Int J Cancer 143: 2150-2160, 2018.

16. Wang K, Li J, Xiong YF, Zeng Z, Zhang X and Li HY: A potential prognostic long noncoding RNA signature to predict recurrence among ER-positive breast cancer patients treated with tamoxifen. Sci Rep 8: 3179, 2018

17. Sun M, Wu D, Zhou K, Li H, Gong X, Wei Q, Du M, Lei P, Zha J, Zhu H, et al: An eight-lncRNA signature predicts survival of breast cancer patients: A comprehensive study based on weighted gene co-expression network analysis and competing endogenous RNA network. Breast Cancer Res Treat 175: 59-75, 2019.
18. Sabatier R, Finetti P, Adelaide J, Guille A, Borg JP, Chaffanet M, Lane L, Birnbaum D and Bertucci F: Down-regulation of ECRG4, a candidate tumor suppressor gene, in human breast cancer. PLoS One 6: e27656, 2011

19. Sabatier R, Finetti P, Cervera N, Lambaudie E, Esterni B, Mamessier E, Tallet A, Chabannon C, Extra JM, Jacquemier J, et al: A gene expression signature identifies two prognostic subgroups of basal breast cancer. Breast Cancer Res Treat 126: 407-420, 2011.

20. Jézéquel P, Loussouarn D, Guérin-Charbonnel C, Campion L, Vanier A, Gouraud W, Lasla H, Guette C, Valo I, Verrièle V and Campone M: Gene-expression molecular subtyping of triple-negative breast cancer tumours: Importance of immune response. Breast Cancer Res 17: 43, 2015.

21. Li Y, Zou L, Li Q, Haibe-Kains B, Tian R, Li Y, Desmedt C, Sotiriou C, Szallasi Z, Iglehart JD, et al: Amplification of LAPTM4B and YWHAZ contributes to chemotherapy resistance and recurrence of breast cancer. Nat Med 16: 214-218, 2010.

22. Guo JC, Wu Y, Chen Y, Pan F, Wu ZY, Zhang JS, Wu JY, $\mathrm{Xu} \mathrm{XE}, \mathrm{Zhao} \mathrm{JM}, \mathrm{Li}$ EM, et al: Protein-coding genes combined with long noncoding RNA as a novel transcriptome molecular staging model to predict the survival of patients with esophageal squamous cell carcinoma. Cancer Commun (Lond) 38: 4 , 2018.

23. Zapata I, Moraes LE, Fiala EM, Zaldivar-Lopez S, Couto CG, Rowell JL and Alvarez CE: Risk-modeling of dog osteosarcoma genome scans shows individuals with Mendelian-level polygenic risk are common. BMC Genomics 20: 226, 2019.

24. Yu G, Wang LG, Han Y and He QY: clusterProfiler: An R package for comparing biological themes among gene clusters. OMICS 16: 284-287, 2012.

25. Yu G, Wang LG, Yan GR and He QY: DOSE: An R/Bioconductor package for disease ontology semantic and enrichment analysis. Bioinformatics 31: 608-609, 2015.

26. Liu W: LncRNA LINC-PINT inhibits cancer cell proliferation, invasion, and migration in osteosarcoma by downregulating miRNA-21. Cancer Biother Radiopharm 34: 258-263, 2019.

27. Marín-Béjar O, Mas AM, González J, Martinez D, Athie A, Morales X, Galduroz M, Raimondi I, Grossi E, Guo S, et al: The human lncRNA LINC-PINT inhibits tumor cell invasion through a highly conserved sequence element. Genome Biol 18: $202,2017$.

28. Zou Z, Ma T, He X, Zhou J, Ma H, Xie M, Liu Y, Lu D, Di S and Zhang Z: Long intergenic non-coding RNA 00324 promotes gastric cancer cell proliferation via binding with $\mathrm{HuR}$ and stabilizing FAM83B expression. Cell Death Dis 9: 717, 2018.

29. Wang S, Zhang S, He Y, Huang X, Hui Y and Tang Y: HOXA11-AS regulates JAK-STAT pathway by miR-15a-3p/STAT3 axis to promote the growth and metastasis in liver cancer. J Cell Biochem 120: 15941-15951, 2019.

30. Chen X, Li X, Fan Z, Li J, Xie Y, Wang T and Ouyang T: Ultrasound as a replacement for physical examination in clinical staging of axillary lymph nodes in breast cancer patients. Thorac Cancer 11: 48-54, 2020.

31. Shui R, Liang X, Li X, Liu Y, Li H, Xu E, Zhang Z, Lian Y, Guo S, Yao M, et al: Hormone receptor and human epidermal growth factor receptor 2 detection in invasive breast carcinoma: A retrospective study of 12,467 patients from 19 chinese representative clinical centers. Clin Breast Cancer, Aug 23, 2019 (Epub ahead of print).

32. Montes P, Bernal M, Campo LN, González-Ramírez AR, Jiménez P, Garrido P, Jurado M, Garrido F, Ruiz-Cabello F and Hernández F: Tumor genetic alterations and features of the immune microenvironment drive myelodysplastic syndrome escape and progression. Cancer Immunol Immunother 68: 2015-2027, 2019

33. Jiang L, Zhao XH, Mao YL, Wang JF, Zheng HJ and You QS: Long non-coding RNA RP11-468E2.5 curtails colorectal cancer cell proliferation and stimulates apoptosis via the JAK/STAT signaling pathway by targeting STAT5 and STAT6. J Exp Clin Cancer Res 38: 465, 2019.

34. Cao W, Gao W, Zheng P, Sun X and Wang L: Medroxyprogesterone acetate causes the alterations of endoplasmic reticulum related mRNAs and lncRNAs in endometrial cancer cells. BMC Med Genomics 12: 163, 2019.

35. Verhoeven RJA, Tong S, Mok BW, Liu J, He S, Zong J, Chen Y, Tsao SW, Lung ML and Chen H: Epstein-barr virus BART long non-coding RNAs function as epigenetic modulators in nasopharyngeal carcinoma. Front Oncol 9: 1120, 2019. 
36. Bao G, Huang J, Pan W, Li X and Zhou T: Long noncoding RNA CERS6-AS1 functions as a malignancy promoter in breast cancer by binding to IGF2BP3 to enhance the stability of CERS6 mRNA. Cancer Med 9: 278-289, 2020.

37. Tang T, Guo C, Xia T, Zhang R, Zen K, Pan Y and Jin L: LncCCAT1 promotes breast cancer stem cell function through activating WNT/ $\beta$-catenin Signaling. Theranostics 9: 7384-7402, 2019.

38. Xue X, Yang YA, Zhang A, Fong KW, Kim J, Song B, Li S, Zhao JC and Yu J: LncRNA HOTAIR enhances ER signaling and confers tamoxifen resistance in breast cancer. Oncogene 35: 2746-2755, 2016.

39. Wang Y,Zhou J, Wang Z, Wang P and Li S: Upregulation of SOX2 activated LncRNA PVT1 expression promotes breast cancer cell growth and invasion. Biochem Biophys Res Commun 493: 429-436, 2017.

40. Li Z, Hou P, Fan D, Dong M, Ma M, Li H, Yao R, Li Y, Wang G, Geng P, et al: The degradation of EZH2 mediated by lncRNA ANCR attenuated the invasion and metastasis of breast cancer. Cell Death Differ 24: 59-71, 2017.

41. Siow ZR, De Boer RH, Lindeman GJ and Mann GB: Spotlight on the utility of the Oncotype $\mathrm{DX}^{\circledR}$ breast cancer assay. Int J Womens Health 10: 89-100, 2018.

42. Tan IB and Tan P: Genetics: Genetics: An 18-gene signature $\left(\right.$ ColoPrint $\left.^{\circledR}\right)$ for colon cancer prognosis. Nat Rev Clin Oncol 8: 131-133, 2011.

43. He Y, Li X, Meng Y, Fu S, Cui Y, Shi Y and Du H: A prognostic 11 long noncoding RNA expression signature for breast invasive carcinoma. J Cell Biochem 120: 16692-16702, 2019.

44. Guo W, Wang Q, Zhan Y, Chen X, Yu Q, Zhang J, Wang Y, Xu XJ and Zhu L: Transcriptome sequencing uncovers a three-long noncoding RNA signature in predicting breast cancer survival. Sci Rep 6: 27931, 2016.

45. Zhao $\mathrm{K}$, Wang $\mathrm{M}$, Kang $\mathrm{H}$ and $\mathrm{Wu} \mathrm{A}$ : A prognostic five long-noncoding RNA signature for patients with rectal cancer. J Cell Biochem, Nov 10, 2019 (Epub ahead of print).

46. Lv J, Guo Y, Yan L, Lu Y, Liu D and Niu J: Development and validation of a five-lncRNA signature with prognostic value in colon cancer. J Cell Biochem, Nov 3, 2019 (Epub ahead of print).
47. Zhang W, Yuan W, Song J, Wang S and Gu X: LncRNA CPS1-IT1 suppresses EMT and metastasis of colorectal cancer by inhibiting hypoxia-induced autophagy through inactivation of HIF-1 $\alpha$. Biochimie 144: 21-27, 2018.

48. Zhang W, Yuan W, Song J, Wang S and Gu X: LncRna CPS1-IT1 suppresses cell proliferation, invasion and metastasis in colorectal cancer. Cell Physiol Biochem 44: 567-580, 2017.

49. Wang YS, Ma LN, Sun JX, Liu N and Wang H: Long non-coding RNA CPS1-IT1 is a positive prognostic factor and inhibits epithelial ovarian cancer tumorigenesis. Eur Rev Med Pharmacol Sci 21: 3169-3175, 2017.

50. Wang TH, Yu CC, Lin YS, Chen TC, Yeh CT, Liang KH, Shieh TM, Chen CY and Hsueh C: Long noncoding RNA CPS1-IT1 suppresses the metastasis of hepatocellular carcinoma by regulating HIF-1 $\alpha$ activity and inhibiting epithelial-mesenchymal transition. Oncotarget 7: 43588-43603, 2016.

51. Rong L, Zhao R and Lu J: Highly expressed long non-coding RNA FOXD2-AS1 promotes non-small cell lung cancer progression via $\mathrm{Wnt} / \beta$-catenin signaling. Biochem Biophys Res Commun 484: 586-591, 2017

52. Chen G, Sun W, Hua X, Zeng W and Yang L: Long non-coding RNA FOXD2-AS1 aggravates nasopharyngeal carcinoma carcinogenesis by modulating miR-363-5p/S100A1 pathway. Gene 645: 76-84, 2018.

53. Bao J, Zhou C, Zhang J, Mo J, Ye Q, He J and Diao J: Upregulation of the long noncoding RNA FOXD2-AS1 predicts poor prognosis in esophageal squamous cell carcinoma. Cancer Biomark 21: 527-533, 2018.

54. Li W, Jia G, Qu Y, Du Q, Liu B and Liu B: Long non-coding RNA (LncRNA) HOXA11-AS promotes breast cancer invasion and metastasis by regulating epithelial-mesenchymal transition. Med Sci Monit 23: 3393-3403, 2017.

55. Pan ZH, Guo XQ, Shan J and Luo SX: LINC00324 exerts tumor-promoting functions in lung adenocarcinoma via targeting miR-615-5p/AKT1 axis. Eur Rev Med Pharmacol Sci 22: 8333-8342, 2018.

56. Zhang H, Cai Y, Zheng L, Zhang Z, Lin X and Jiang N: LncRNA BISPR promotes the progression of thyroid papillary carcinoma by regulating miR-21-5p. Int $\mathrm{J}$ Immunopathol Pharmacol 32: 2058738418772652, 2018 